\title{
COVID-19: How to Prepare for the Pandemic?
}

\author{
Rakesh Lodha $^{1}$ - S. K. Kabra ${ }^{1}$
}

Received: 1 April 2020 / Accepted: 2 April 2020 / Published online: 13 April 2020

(C) Dr. K C Chaudhuri Foundation 2020

\section{Introduction}

The world is facing yet another pandemic- that of a novel Coronavirus infection. The epicenter of pandemic was Wuhan in China and by this time the infection has involved nearly all the countries, affecting high-income as well as the low-income countries. Though it is transmitted mainly by droplets, secondary infection rates are high. Currently, about $20 \%$ of those infected have significant problems and need hospitalization and $5 \%$ of total infected may develop acute severe respiratory illness, and need intensive care including mechanical ventilation; the latter group has high case fatality, especially among elderly and those with comorbidities $[1,2]$. In the absence of proven effective antiviral drugs, all patients need symptomatic and supportive treatment. Acute respiratory distress syndrome needs aggressive ventilatory support and also multiple organ support. Patients may need intensive care for weeks. Intensive care beds are limited and even developed countries are facing with shortage of ICU beds in view of sudden surge in the numbers of infected cases. Most patients with severe disease need ventilatory support for few weeks and there is shortage of ventilators. Health care personnel (HCP) have been reported to have high infection rate; in the absence of specific antiviral therapy and potential for more severe disease in HCP (possibly due to high inoculum of infection/ multiple exposures) [3], adequate personal protective equipment (PPE) is necessary for all HCP to prevent infection in them. Most countries are facing major shortage of PPEs [4].

We need to prepare ourselves about how best we can handle this situation.

\section{S. K. Kabra}

skkabra@live.in

1 Department of Pediatrics, All India Institute of Medical Sciences, Ansari Nagar, New Delhi, India

\section{Assess the Existing Health Capacity and Anticipate the Surge}

In the current crises, it is apparent that there is likely to be a wide gap between demand and availability of hospital beds, intensive care facilities, ventilators, trained human resources, disposables, PPEs, capacity to handle other associated problems that may arise from this pandemic. For example, in India, even if we consider $0.1 \%$ of population gets infected over next $60 \mathrm{~d}$, and $5 \%$ of those infected need critical care, we need ICU beds for 65,000 people. An average duration of mechanical ventilation of $15 \mathrm{~d}$, which translates into approximately 975,000 ventilator days. If this surge in epidemic is spread over $60 \mathrm{~d}$, we need approximately 16,200 ventilators on a given day [total infection 1,300,000, hospitalization (20\%) 260,000, ICU 65000]. While this is a simplistic calculation which assumes $0.1 \%$ infection rate, with higher infection rate of $1 \%$, the numbers of ventilators required will be approximately 162,000 . As of now India does not have that capacity [5]. Similarly, the capacity for intensive care in the country, including that in public health system is limited. While ventilators may still be sourced/ purchased, the necessary staffing will still be a major limiting factor. Therefore, it is clear that even if we prepare ourselves for the disaster there will be wide gap in demand and availability. In such a situation, facilities may not be in a position to provide appropriate medical care to all those in need, particularly if we have a massive outbreak similar to other countries such as Italy, Spain, Iran and United States.

In the scenario of setting up a fully functional ICU to deal with the sickest of COVID patients, policy makers should ensure that resource allocation for developing intensive care would not adversely affect the resource allocation for care of moderately ill patients, whose numbers are substantial and the outcomes in these with appropriate management are excellent. There is need to develop innovative strategies for health care by multiple means, with particular emphasis on prevention.

All medical fraternity has to work together in this hour of crisis to flatten the epidemic curve. With help of public health experts, we may try to implement population-based 
interventions like social distancing in general public, quarantine of those exposed to known cases of COVID-19 and isolation of affected individuals.

\section{Generating Resources}

Looking at the infectiousness of SARS nCoV, there is a need to segregate services for COVID and non-COVID patients. If same facility is used for both the groups, there will be chance of infection getting disseminated through patients and their attendants visiting the hospital [6]. This strategy is heavily dependent on accurate testing with a short turn-around time. We need relatively well equipped hospitals for acute care, which can be converted to COVID hospitals. The COVID hospitals will have a higher proportion of bed strength allocated to ICU and high dependency units. There will be a need for various equipments such as ventilators, multi-parameter monitors, pulse oximeters, non-invasive ventilators, infusion pumps, etc. as well need for adequate supply of oxygen, preferably a high pressure oxygen source. While there may be a shortfall in equipment, having adequate oxygen supplies is also likely to be an important challenge. It's also a challenge to procure suitable, appropriate quality consumables and accessories in adequate quantities. It may be worthwhile enhancing the care of moderately ill patients before scaling up the ICU beds where the expertise to run ICUs is limited.

Another area of work could be developing infrastructure to reduce the burden on health facilities due to mildly symptomatic COVID patients; these will be approximately $80 \%$ of all infected cases. There could be platforms developed to facilitate evaluation and management of mildly symptomatic patients. This round the clock service should have options for a video consultation, and follow up. This will prevent a large number of visits by patients and their attendants to the hospital [7]. Limited experience in children suggests that COVID-19 infection is less common and less severe. Therefore, a child with severe acute respiratory illness (SARI) is less likely to have COVID as an etiology; so, these children (awaiting results of infection) should preferably be kept in separate isolation to reduce exposure from adult patients. It is suggested that approximately $5 \%$ of ICU beds be earmarked for children and about $10 \%$ in HDU/ wards. Additionally, adequate arrangements will be required for an attendant (parent/ relative) to stay with the child and also adequate PPE for the attendant should be provided.

When health care facilities get overwhelmed with COVID19 , it is also important to make sure that non-COVID-19 emergencies continue to get full attention. Similarly, patients with chronic illnesses who are on long term treatment should continue to get their medications and consultations. General public may be afraid or find it difficult to come and seek medical help in case of emergency. It is important to ensure that private /public health care facilities provide care to nonCOVID-19 emergencies and help/guide patients in getting their regular medications. Individuals getting their medications from government establishments (anti-tuberculosis drugs, antiretroviral drugs, etc.) should continue to get services without interruption. Patients under follow up with doctors may get consultation by teleconferencing or other electronic media during the period of lockdown.

\section{Development of Human Resource}

For efficient care of patients with COVID-19, and protecting HCPs from infection, it is very important to train all HCPsdoctors, nurses, technicians, ward boys, sanitation workers, and other support staff for each hospital rapidly. Each hospital has trained persons in different departments. Existing resources can be utilized by making a virtual Disaster Management Department with people from different departments/ facilities. Broadly, it may include personnel from Hospital Administration, Public Health, Anesthesiology, Medicine, Geriatrics, Pediatrics, Pulmonary Medicine, Critical Care Medicine, Microbiology, Nursing, etc. Under supervision of an experienced HCP, small teams covering different domains can be made to help in developing services in a short time. Each team can meet and discuss about their needs, pooling of resources and developing effective communication with each other by electronic media. This department may pool all resources and redistribute as per the needs [8].

The key areas for training include prevention of infection among HCPs, appropriate use of PPEs, clinical management of patients both in out-patient and in-patient services, oxygen therapy, respiratory support, management of critically ill patients, handling various equipments, etc. The training can rapidly be scaled up using telemedicine; having content online. It may be useful to have teleconsultation facilities to support medical teams in hospitals with newly trained staff. Medical colleges can adopt district hospitals and smaller hospitals, and provide them support.

To manage a diverse pool of staff with varied levels of training, smart management skills will be required.

\section{PPEs, Recycling, Innovations}

There are reports from all over the world about shortage of PPEs. Manufacture of PPEs and also there raw materials was heavily dependent on China; after the beginning of the pandemic in China, these supplies have been disrupted and the capacity to manufacture and supply is currently extremely limited, particularly in the setting of a massive increase in the demand. The authorities must make all possible efforts 
to make high quality PPE available for the HCPs; this should be the highest priority area. Conventionally, for best results, all PPEs should be properly disposed off after single use. In view of wide gap between demand and supply, some innovations and possible reuse of PPEs is required. There are some reports of reuse of various PPE items (N 95 masks, face shield, etc.) by using sterilizing techniques of hydrogen peroxide vapor [9]. However, these should only be considered as back-up options and not lead to complacency in making adequate PPE available.

At the same time there is a need to innovate to provide protection for the HCPs; these could include do-it-yourself face shields, masks, etc.

\section{Care of HCPs}

Health care personnel are the front-line people to manage the patients in this pandemic. They are at a significant risk of contracting infection and falling sick. It is important that each hospital prepares appropriate facilities for their treatment; this in fact may take a priority over management of non-HCP COVID-19 patients [10]. It is equally important to keep them motivated and keep their morale high. It is important to keep duty hours short. Health care workers taking care of infected persons, may face conflict between professional duty and duty towards their family members. There should be a provision to handle such situations including professional help and rotation of HCP. To have an adequate pool, training of staff from all the areas of medicine is a must [11].

\section{Management with Limited Resources}

Various bodies in the affected countries have come up with management guidelines; in absence of specific antiviral treatment, these mainly focus on supportive care and appropriate organ support in intensive care units. Many of our facilities may not be able to provide care as per these guidelines; we have to be realistic and provide best possible care using protocols that are best suited to our resources and requirement. As highlighted above, even in the absence of high end intensive care, a large number of lives could be saved by providing care to moderately sick patients. Given the huge diversity in the levels of health facilities in our country, multiple such protocols may be needed.

\section{Post Acute Care, Convalescent Care}

It is equally important think of care of patients who have recovered from acute problems but have problems due to comorbidities. Before sending them home, it is important to take care of their needs, otherwise mishaps may happen after discharge. Equally important is to think of providing care for various psychological problems following traumatic experience [12].

\section{Resolution of Administrative and Ethical Challenges}

When there is a wide gap between demand and supply, there may be lot of pressure for getting tests done, admission in ICU, getting ventilatory support, etc. on the teams managing the screening and triage area, working in ICUs, laboratories, etc. Similarly, there may be some conflict about starting or removing life support for critically sick patients, particularly in the very old and those with multiple comorbidities [10]. A treating team alone may not be in a position to take decisions. To overcome these sensitive issues, a high-powered committee who is not directly involved in patient care can resolve these issues.

\section{Research Priorities and Enhancing Research}

As this is a new disease with many unknowns, it is imperative that health care facilities identify research priorities to answer some of the questions they faced during development of facilities as well as they face during patient management. A carefully developed data management of all the patients may answer some of the epidemiological questions. It is important that each health care facility records all the data of screening, triage, management, etc. in a structured manner. The availability of information technology experts in the country may be useful in developing these.

\section{Conclusions}

The rapid spread of the SARS nCoV 2 pandemic has brought the world to a standstill and imposed multiple serious challenges in the developed world; these challenges are likely to be even greater in countries such as India if there is a surge in infected cases. In the setting of weak health system, the preparedness to face the onslaught has to be ramped up rapidly. At the same time, there has to be judicious use of the available resources with a high priority for safety of the health care professionals- the front-line warriors in this situation. In absence of specific antiviral treatments as of now, supportive care for the diseased and use of multiple preventive strategies appear to be the key to containing the pandemic.

Authors' Contributions RL, SKK: Involved in literature search and writing of manuscript. RL will act as guarantor for the paper. 


\section{Compliance with Ethical Standards}

Conflict of Interest None.

\section{References}

1. Zhou F, Yu T, Du R, et al. Clinical course and risk factors for mortality of adult inpatients with COVID-19 in Wuhan, China: a retrospective cohort study. Lancet. 2020;395:1054-62.

2. Weiss P, Murdoch DR. Clinical course and mortality risk of severe COVID-19. Lancet. 2020;395:1014-5.

3. Malhotra N, Gupta N, Ish S, Ish P. COVID-19 in intensive care. Some necessary steps for health care workers. Monaldi Arch Chest Dis. 2020;90. https://doi.org/10.4081/monaldi.2020.1284.

4. World Health Organization. Shortage of personal protective equipment endangering health workers worldwide. WHO, Geneva. 3 March 2020. Available at: https:/www.who.int/news-room/detail/ 03-03-2020-shortage-of-personal-protective-equipmentendangering-health-workers-worldwide. Accessed 1 April 2020.

5. Bhutta ZA, Basnyat B, Saha S, Laxminarayan R. Covid-19 risks and response in South Asia. BMJ. 2020;368:m1190.
6. Hick JL, Biddinger PD. Novel coronavirus and old lessons Preparing the health system for the pandemic. N Engl J Med. 2020. https://doi.org/10.1056/NEJMp2005118.

7. Hollander JE, Carr BG. Virtually perfect? Telemedicine for Covid19. N Engl J Med. 2020. https://doi.org/10.1056/NEJMp2003539.

8. Agarwal A, Nagi N, Chatterjee P, et al. Guidance for building a dedicated health facility to contain the spread of the 2019 novel coronavirus outbreak. Indian J Med Res. 2020. https://doi.org/10. 4103/ijmr.IJMR_518_20.

9. Kampf G, Todt D, Pfaender S, Steinmann E. Persistence of coronaviruses on inanimate surfaces and their inactivation with biocidal agents. J Hosp Infect. 2020;104:246-51.

10. Emanuel EJ, Persad G, Upshur R, et al. Fair allocation of scarce medical resources in the time of Covid-19. N Engl J Med. 2020. https://doi.org/10.1056/NEJMsb2005114.

11. Huh S. How to train the health personnel for protecting themselves from novel coronavirus (COVID-19) infection during their patient or suspected case care. J Educ Eval Health Prof. 2020;17:10.

12. Grabowski DC, Joynt Maddox KE. Postacute care preparedness for COVID-19: Thinking ahead. JAMA. 2020. https://doi.org/10.1001/ jama.2020.4686.

Publisher's Note Springer Nature remains neutral with regard to jurisdictional claims in published maps and institutional affiliations. 